\title{
Ultrasonographic features of focal cortical dysplasia and their relevance for epilepsy surgery
}

\author{
Kevin Akeret, MD, ${ }^{1}$ David Bellut, MD, ${ }^{1}$ Hans-Jürgen Huppertz, MD, ${ }^{4}$ Georgia Ramantani, MD, PhD, ${ }^{3,4}$ \\ Kristina König, MD, ${ }^{4}$ Carlo Serra, MD, ${ }^{1}$ Luca Regli, MD, ${ }^{1}$ and Niklaus Krayenbühl, MD ${ }^{1,2}$ \\ ${ }^{1}$ Department of Neurosurgery, Clinical Neuroscience Center, University Hospital Zurich, University of Zurich; '2Division of \\ Pediatric Neurosurgery, University Children's Hospital, Zurich; ${ }^{3}$ Division of Pediatric Neurology, University Children's Hospital, \\ Zurich; and ${ }^{4}$ Swiss Epilepsy Clinic, Klinik Lengg AG, Zurich, Switzerland
}

OBJECTIVE Surgery has proven to be the best therapeutic option for drug-refractory cases of focal cortical dysplasia (FCD)-associated epilepsy. Seizure outcome primarily depends on the completeness of resection, rendering the intraoperative FCD identification and delineation particularly important. This study aims to assess the diagnostic yield of intraoperative ultrasound (IOUS) in surgery for FCD-associated drug-refractory epilepsy.

METHODS The authors prospectively enrolled 15 consecutive patients with drug-refractory epilepsy who underwent an IOUS-assisted microsurgical resection of a radiologically suspected FCD between January 2013 and July 2016. The findings of IOUS were compared with those of presurgical MRI postprocessing and the sonographic characteristics were analyzed in relation to the histopathological findings. The authors investigated the added value of IOUS in achieving completeness of resection and improving postsurgical seizure outcome.

RESULTS The neurosurgeon was able to identify the dysplastic tissue by IOUS in all cases. The visualization of FCD type I was more challenging compared to FCD || and the demarcation of its borders was less clear. Postsurgical MRI showed residual dysplasia in 2 of the 3 patients with FCD type I. In all FCD type II cases, IOUS allowed for a clear intraoperative visualization and demarcation, strongly correlating with presurgical MRI postprocessing. Postsurgical MRI confirmed complete resection in all FCD type II cases. Sonographic features correlated with the histopathological classification of dysplasia (sonographic abnormalities increase continuously in the following order: FCD IA/IB, FCD IC, FCD IIA, FCD IIB). In 1 patient with IOUS features atypical for FCD, histopathological investigation showed nonspecific gliosis. CONCLUSIONS Morphological features of FCD, as identified by IOUS, correlate well with advanced presurgical imaging. The resolution of IOUS was superior to MRI in all FCD types. The appreciation of distinct sonographic features on IOUS allows the intraoperative differentiation between FCD and non-FCD lesions as well as the discrimination of different histological subtypes of FCD. Sonographic demarcation depends on the underlying degree of dysplasia. IOUS allows for more tailored resections by facilitating the delineation of the dysplastic tissue.

https://thejns.org/doi/abs/10.3171/2018.6.FOCUS18221

KEYWORDS focal cortical dysplasia; malformations of cortical development; intraoperative ultrasound; intraoperative sonography; epilepsy surgery

$\mathrm{E}$ PILEPSY affects more than 50 million people worldwide. ${ }^{54}$ Approximately one-third of patients show inadequate seizure control with antiepileptic drugs (AEDs). ${ }^{31,32}$ In children, focal cortical dysplasia (FCD) is the most frequent underlying pathology, accounting for more than $50 \%$ of cases. ${ }^{4}$ FCD was first described by Taylor et al. in $1971^{50}$ and its classification has since undergone several modifications. ${ }^{37,40,49}$ In 2011, the first international consensus classification was released, differentiating 3 main categories of FCD and 9 subtypes, ${ }^{5}$ which have been widely used ever since. ${ }^{39}$ In FCD-associated epilepsy, seizure onset usually occurs in early childhood ${ }^{39,42,53}$ and the clinical course is often severe, with high seizure frequency, high rates of status epilepticus, and poor AED response..$^{18}$ FCDs often escape detection on conventional MRI., ${ }^{9,13,27,30}$ However, recent technological advances, particularly

ABBREVIATIONS AED = antiepileptic drug; ECoG = electrocorticography; EEG = electroencephalography; FCD = focal cortical dysplasia; ILAE = International League Against Epilepsy; IOUS = intraoperative ultrasound. 
TABLE 1. Overview of available literature regarding the use of IOUS in patients with FCD

\begin{tabular}{|c|c|c|c|c|c|c|}
\hline \multirow[b]{2}{*}{ Authors \& Year } & \multirow{2}{*}{$\begin{array}{l}\text { Total } \\
\text { Cases }\end{array}$} & \multirow{2}{*}{$\begin{array}{l}\text { FCD } \\
\text { Type }\end{array}$} & \multicolumn{3}{|c|}{ IOUS } & \multirow{2}{*}{$\begin{array}{l}\text { Sz Outcome } \\
\text { (Engel class) }\end{array}$} \\
\hline & & & Identification & Clear Demarcation & Features & \\
\hline Miller et al., 2008 & 1 & IIB & Yes & Yes & Clear HE & 1 \\
\hline \multirow[t]{5}{*}{ Miller et al., 2011} & 5 & IA & Yes & No & Faint HE & II \\
\hline & & IB & No & No & None & II \\
\hline & & $\| B^{*}$ & Yes & Yes & Clear HE & I \\
\hline & & II & Yes & Yes & Clear HE & II \\
\hline & & II & Yes & Yes & Clear HE & II \\
\hline Lee et al., 2014 & 1 & $\| A$ & Yes & Yes & Clear HE & I \\
\hline
\end{tabular}

MRI postprocessing techniques such as morphometric analysis, ${ }^{23,24,52}$ curvilinear reformatting, ${ }^{25}$ and quantitative FLAIR analysis ${ }^{20}$ increasingly allow for FCD detection and, consequently, for resection.

Surgery is the most promising intervention to achieve seizure control in FCD-associated drug-resistant epilepsy. The reported postsurgical freedom from seizures ranges widely-between $33 \%$ and $90 \%, 9,10,12,15,21,22,47-49,51$ depending to a great extent on presurgical candidate selection. The identification of the precise extent of the dysplastic cortical region and, consequently, its complete resection has proven to be the single most important prognostic factor for postsurgical seizure freedom. . $2,15,26,29,41,43$ Seizures may originate from the center as well as the periphery of the FCD, and the regions of most severe dysplasia do not necessarily overlap with the regions of highest epileptogenicity. Thus, complete FCD resection, as opposed to a resection of the central part of the lesion or of its most dysplastic areas, is crucial for surgical success. ${ }^{6}$ Intraoperative tools currently used to define lesion borders include electrocorticography (ECoG), neuronavigation, and intraoperative MRI, as well as visual and tactile information. However, all of these tools have their intrinsic limitations.

Intraoperative ultrasound (IOUS) is a promising tool for intraoperative anatomical guidance, particularly regarding the demarcation of lesion borders, thereby enabling more complete resection. The efficacy of IOUS in surgery for FCD-associated drug-resistant epilepsy has been indicated by Miller et al. in a case report in $2008^{35}$ and in a retrospective 5-patient series in $2011,{ }^{36}$ as well as by Lee et al. in a case report in $2014^{33}$ (Table 1). Our study aimed to extend previous research in this field by systematically and prospectively assessing the diagnostic yield of IOUS in surgery for FCD-associated drug-resistant epilepsy in a larger cohort. For this purpose, we compared IOUS findings with those of presurgical postprocessed MRI, and we analyzed IOUS features in relation to the histologically confirmed FCD subtype. We investigated the added value of IOUS in improving completeness of resection and, thus, postsurgical seizure outcomes.

\section{Methods \\ Patient Selection}

We prospectively enrolled 15 consecutive patients with drug-resistant focal epilepsy associated with a radiologically suspected FCD who underwent epilepsy surgery in our institution between January 2013 and July 2016. All patients received comprehensive presurgical evaluation according to a standard protocol in the Swiss Epilepsy Center at Klinik Lengg in Zurich, the University Hospital Zurich, and/or the University Children's Hospital Zurich and were referred for surgical treatment by an interdisciplinary case management board. All patients and/or their parents gave their written informed consent for the participation in this study. The study was approved by the local ethics committee.

\section{Presurgical Evaluation}

\section{Demographics and Epilepsy Course}

Systematically and prospectively collected data included demographics (sex, age at surgery); epilepsy history (age at epilepsy onset, duration of epilepsy to surgery, seizure classification according to the International League Against Epilepsy [ILAE] $2017^{19}$ system); seizure frequency at surgery; presurgical AED treatment; prior epilepsy surgery; presurgical neurological and neuropsychological deficits; and psychiatric comorbidities.

\section{EEG Studies}

All patients underwent long-term scalp electroencephalography (EEG), including ictal recordings. In addition, 9 patients underwent invasive EEG in a preceding, separate surgery, with subdural grid and strip electrodes and/or with stereotactically implanted depth electrodes. Invasive electrode positions were visualized in 3D format according to the postimplantation MRI. ${ }^{28}$

\section{Preoperative MRI}

MRI was visually reviewed regarding the classification and extent of lesions, including the presence of a secondary pathology. In addition, MRI postprocessing techniques (morphometric analysis, ${ }^{24}$ curvilinear reformatting ${ }^{25}$ and quantitative FLAIR analysis ${ }^{20}$ ) were applied to improve FCD detection and delineation. These tools serve to highlight MRI abnormalities suspicious for FCD - such as abnormal gyral patterns, abnormally wide and/or deep sulci, increased cortical thickness, and blurring of the gray-white-matter junction, as well as abnormal cortical 
TABLE 2. Demographic and clinical data according to histological result in 15 patients with FCD-associated epilepsy

\begin{tabular}{|c|c|c|c|c|c|c|c|c|c|c|c|c|}
\hline \multirow{2}{*}{$\begin{array}{l}\text { Histological } \\
\text { Type }\end{array}$} & \multirow[b]{2}{*}{ Sex } & \multirow{2}{*}{$\begin{array}{l}\text { Age at } \\
\text { Op (yrs) }\end{array}$} & \multirow{2}{*}{$\begin{array}{c}\text { Age at Epilepsy } \\
\text { Onset (yrs) }\end{array}$} & \multirow{2}{*}{$\begin{array}{c}\text { Duration of } \\
\text { Epilepsy (yrs) }\end{array}$} & \multicolumn{4}{|c|}{ Sz Classification (ILAE 2017) } & \multirow{2}{*}{$\begin{array}{c}\text { Preop Sz } \\
\text { Frequency (Szs/wk) }\end{array}$} & \multicolumn{3}{|c|}{ Preop Neurological Abnormalities } \\
\hline & & & & & FO-a & FO-ia & $2^{\circ} \mathrm{TC}$ & $\mathrm{SE}$ & & $F$ & NP & $P$ \\
\hline FCD IA & M & 16 & 7 & 9 & Yes & No & No & No & 0.5 & No & Yes & No \\
\hline FCD IB & $\mathrm{F}$ & 44 & 4 & 40 & Yes & Yes & Yes & No & 21 & Yes & Yes & Yes \\
\hline FCD IC & M & 4 & 0.5 & 3.5 & Yes & Yes & No & No & 14 & No & Yes & Yes \\
\hline FCD $\| A$ & M & 15 & 7 & 8 & Yes & Yes & No & No & 0.5 & No & Yes & No \\
\hline FCD $\| A$ & $M$ & 38 & 0.5 & 37.5 & Yes & Yes & Yes & No & 14 & No & Yes & No \\
\hline FCD $\| A$ & $M$ & 1 & 0.5 & 0.5 & Yes & Yes & Yes & Yes & 7 & No & Yes & No \\
\hline FCD $\| A$ & $\mathrm{~F}$ & 15 & 4 & 11 & Yes & Yes & Yes & No & 10 & Yes & Yes & No \\
\hline FCD $\| A$ & $\mathrm{~F}$ & 3 & 0.5 & 2.5 & No & Yes & No & No & 8 & No & Yes & No \\
\hline FCD IIB & $M$ & 34 & 3 & 31 & Yes & Yes & Yes & Yes & 14 & Yes & Yes & Yes \\
\hline FCD IIB & $M$ & 33 & 8 & 25 & No & Yes & Yes & No & 21 & No & Yes & No \\
\hline FCD $\| B$ & $\mathrm{~F}$ & 29 & 15 & 14 & No & Yes & Yes & No & 7 & No & Yes & Yes \\
\hline FCD $\| B$ & $F$ & 1 & 0.5 & 0.5 & Yes & No & No & No & 56 & No & Yes & No \\
\hline FCD IIB & $\mathrm{F}$ & 6 & 4.5 & 1.5 & Yes & Yes & No & No & 7 & No & No & No \\
\hline FCD $\| B$ & $\mathrm{~F}$ & 7 & 2 & 5 & No & Yes & Yes & No & 1 & No & Yes & Yes \\
\hline Gliosis & M & 4 & 0.5 & 3.5 & Yes & Yes & No & No & 70 & No & Yes & No \\
\hline
\end{tabular}

$\mathrm{F}=$ focal; $\mathrm{FO}-\mathrm{a}=$ focal onset, aware; $\mathrm{FO}-\mathrm{ia}=$ focal onset, impaired awareness; $\mathrm{NP}=$ neuropsychological; $\mathrm{P}=$ psychiatric $\mathrm{SE}=$ status epilepticus; $2^{\circ} \mathrm{TC}=$ focal to bilateral tonic clonic.

${ }^{*}$ Duration at time of surgery.

or subcortical signal intensity, including the transmantle sign. These criteria were based on current literature regarding MRI features of FCDs. . $^{2,13,25,30}$

\section{Surgery and Intraoperative Guidance}

Neuronavigation (Stealth Station; Medtronic) was used in all cases. For this purpose, the localization of the lesion, as indicated by postprocessed MRI, was transferred as a bright marker into a 3D T1-weighted image, which in turn was loaded into the neuronavigation system. After craniotomy and dura mater opening, IOUS was applied, allowing for anatomical orientation, morphological comparison to preoperative imaging, and delineation of the lesion. For this high-resolution intraoperative imaging we used an iU22 Ultrasound System with a real-time L15-7 intraoperative probe (7-15 MHz extended frequency with a phased linear array of 128 elements including a $23-\mathrm{mm}$ effective aperture length, probe tip measure $11 \times 31 \mathrm{~mm}$; Philips). ${ }^{8}$ Intraoperative ECoG was performed in all cases. Thus, the final extent of resection finding was based on the postprocessed preoperative MRI, IOUS, and intraoperative ECoG.

\section{Histopathological Evaluation}

Resection specimens were analyzed by the Department of Neuropathology, University Hospital Zurich. To increase diagnostic certainty, an additional histopathological evaluation was performed in the European FCD Reference Center in Erlangen. FCDs were categorized according to the ILAE classification system. ${ }^{3}$

\section{Follow-Up}

Clinical and EEG follow-up was scheduled at 3,6, and 12 months after surgery and then yearly in cases of sei- zure control or at appropriate intervals in cases of seizure recurrence. MRI was performed 3 months after surgery to assess for potential residual dysplasia, including postprocessing evaluations as well as coregistration with presurgical MRI. Epilepsy course, AED tapering, and neurological deficit were noted. The classification of postsurgical seizure outcome was performed according to the Engel classification system. ${ }^{16}$

\section{Data Analysis}

Due to the small sample size, statistical analysis is provided in a descriptive manner.

\section{Results}

\section{Demographics and Epilepsy Course}

Seven female $(47 \%)$ and 8 male $(53 \%)$ patients were included in our study. The demographic data and the presurgical epilepsy course are given in Table 2. Epilepsy onset occurred at a mean of 3.5 years (SD 4.1, range 0.5-15 years), the mean duration from epilepsy to surgery was 12.4 years (SD 13.4, range 0.5-40 years), and the mean age at surgery was 16.7 years (SD 14.5, range 1-44 years). All but 3 patients were experiencing daily seizures at the time of surgery despite multiple AED trials. None of the patients had previously undergone epilepsy surgery. All but one of our patients presented with focal neurological deficit, neuropsychological impairment, or psychiatric comorbidities prior to surgery.

\section{Presurgical Evaluation}

Topographical and morphological features of the suspected dysplastic areas are presented for all patients in Table 3 and for illustrative cases in Figs. 1-3. Blurring of the 


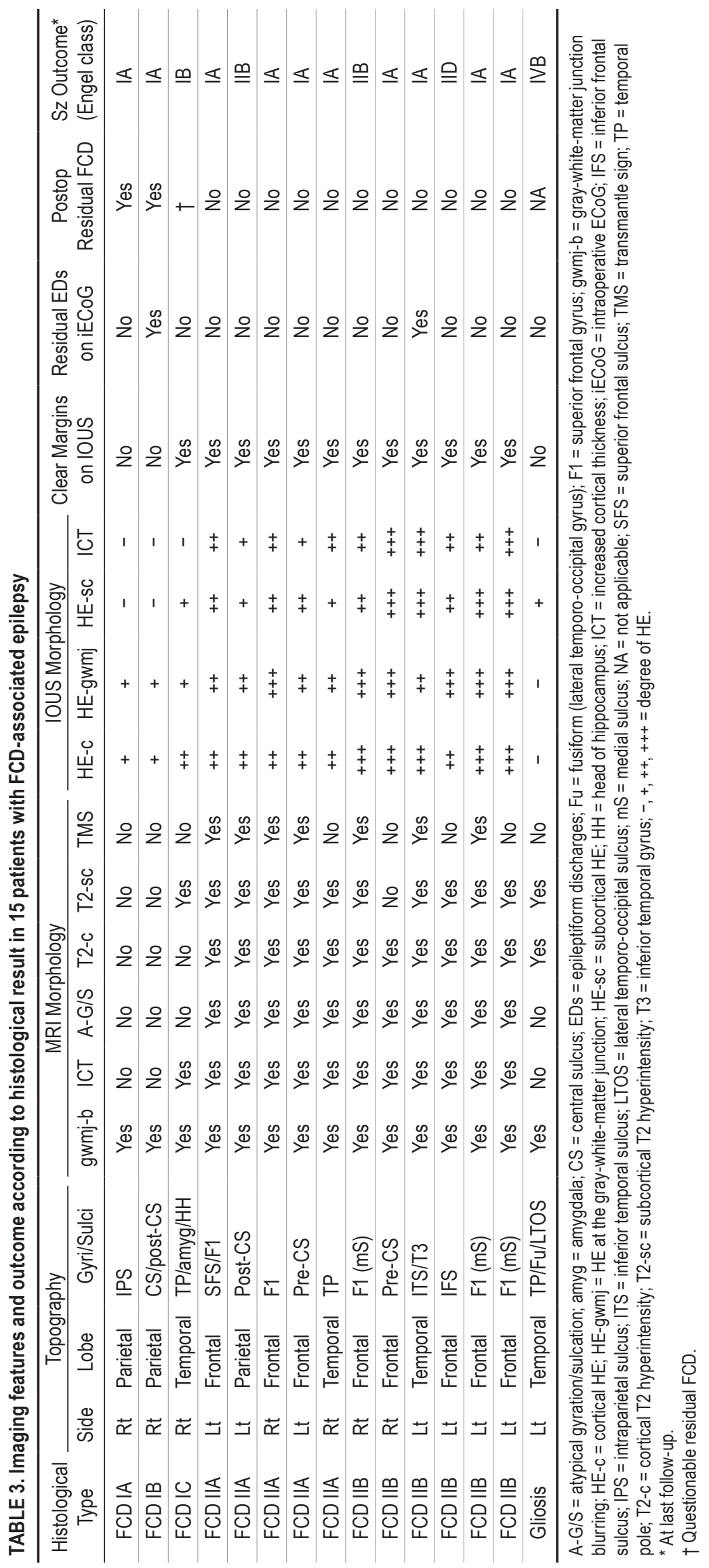




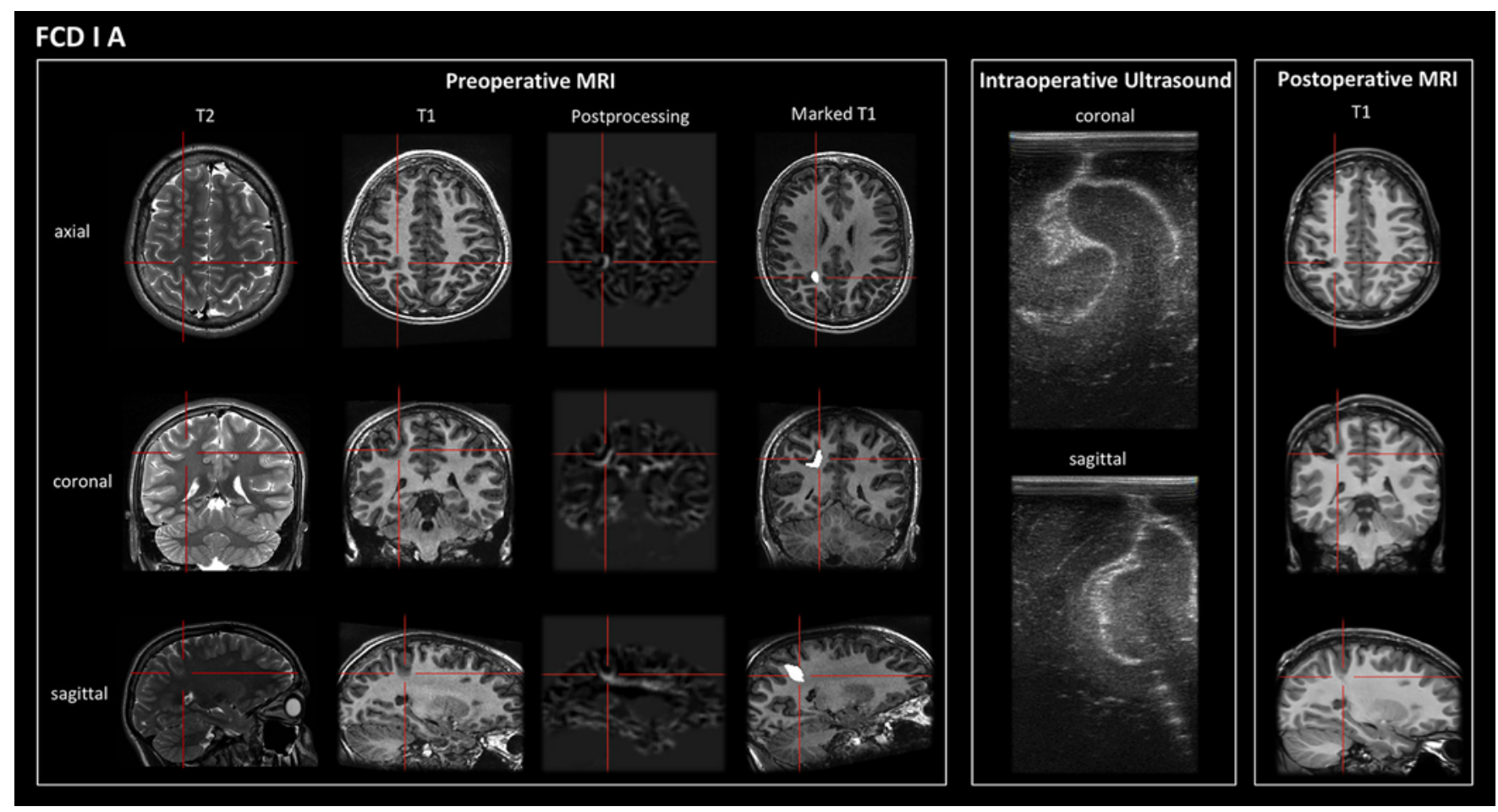

FIG. 1. Examples of pre-, intra-, and postoperative imaging in a patient with type IA FCD in the depth of the right intraparietal sulcus. Preoperative MRI with postprocessing (example sequence shown) allowed us to identify an area of subtle blurring of the gray-white-matter junction suspicious for the presence of FCD. Despite very discrete signal alterations on preoperative MRI, IOUS allowed us to depict the dysplastic area with high resolution by showing hyperechogenicity in the cortex and the underlying gray-white-matter junction. Topographical and morphological features correlated well to preoperative imaging. However, a sharp demarcation of the altered area was not possible, resulting in subtotal resection, with residual dysplasia seen with coregistration between pre- and postoperative MRI.

gray-white-matter junction was the most consistent feature pointing to FCD in our cohort. Radiologically, dual pathology was found in a single patient, who presented with left hippocampal sclerosis in addition to a suspected left mesiotemporal FCD. It should be noted, however, that histopathological investigation did not confirm FCD, only showing nonspecific gliosis in this case.

\section{Intraoperative Ultrasound}

IOUS showed signal abnormalities in the region of suspected FCD in all cases. These sonographic abnormalities presented a high anatomical correlation with the features suspicious for FCD on preoperative MRI (Figs. 1-3), whereas IOUS resolution was superior to MRI in all cases. In addition, the sonographic features of the lesions correlated well with histopathological findings (Fig. 4). In all but one patient, IOUS showed specific and circumscribed signal abnormalities: histopathological investigation confirmed FCD. In a single patient, IOUS showed diffuse signal alterations with a blurry cortical and subcortical hyperechogenicity: histopathological studies revealed only nonspecific gliosis.

In cases with specific and circumscribed signal abnormalities corresponding to histologically verified FCD, the degree of cortical and subcortical signal alterations correlated to the underlying degree of dysplasia; i.e., to the FCD subtype. In the 3 cases of FCD type I, the dysplastic cortical areas showed discrete hyperechogenicity with a blurred border toward the underlying white matter, albeit without any white matter changes. In FCD type I, the dysplastic area was thus identifiable, but the precise demarcation of its borders was challenging. FCD types IA and IB presented with almost identical features in IOUS, whereas FCD type IC was characterized by a higher degree of cortical hyperechogenicity. FCD type II presented with more pronounced sonographic abnormalities, including marked cortical hyperechogenicity, increased cortical thickness, blurred and hyperechogenic gray-white-matter junction, and variably increased white matter signal. These changes were more prominent in FCD type IIB. In FCD type II, the dysplastic area was not only identifiable, but the precise demarcation of its borders was facilitated by these pronounced signal changes. IOUS findings are given in Table 3.

\section{Intraoperative Electrocorticography}

Intraoperative ECoG following the sonographically guided resection revealed residual epileptic discharges in only 2 cases (Table 3 ). In one of these patients, further ECoG-guided resection was limited by the localization of the epileptic discharges within the primary motor area. In the second case the residual epileptic activity in ECoG led to an extension of the previous resection; in repeat ECoG, no unequivocal epileptic discharges were noted. 


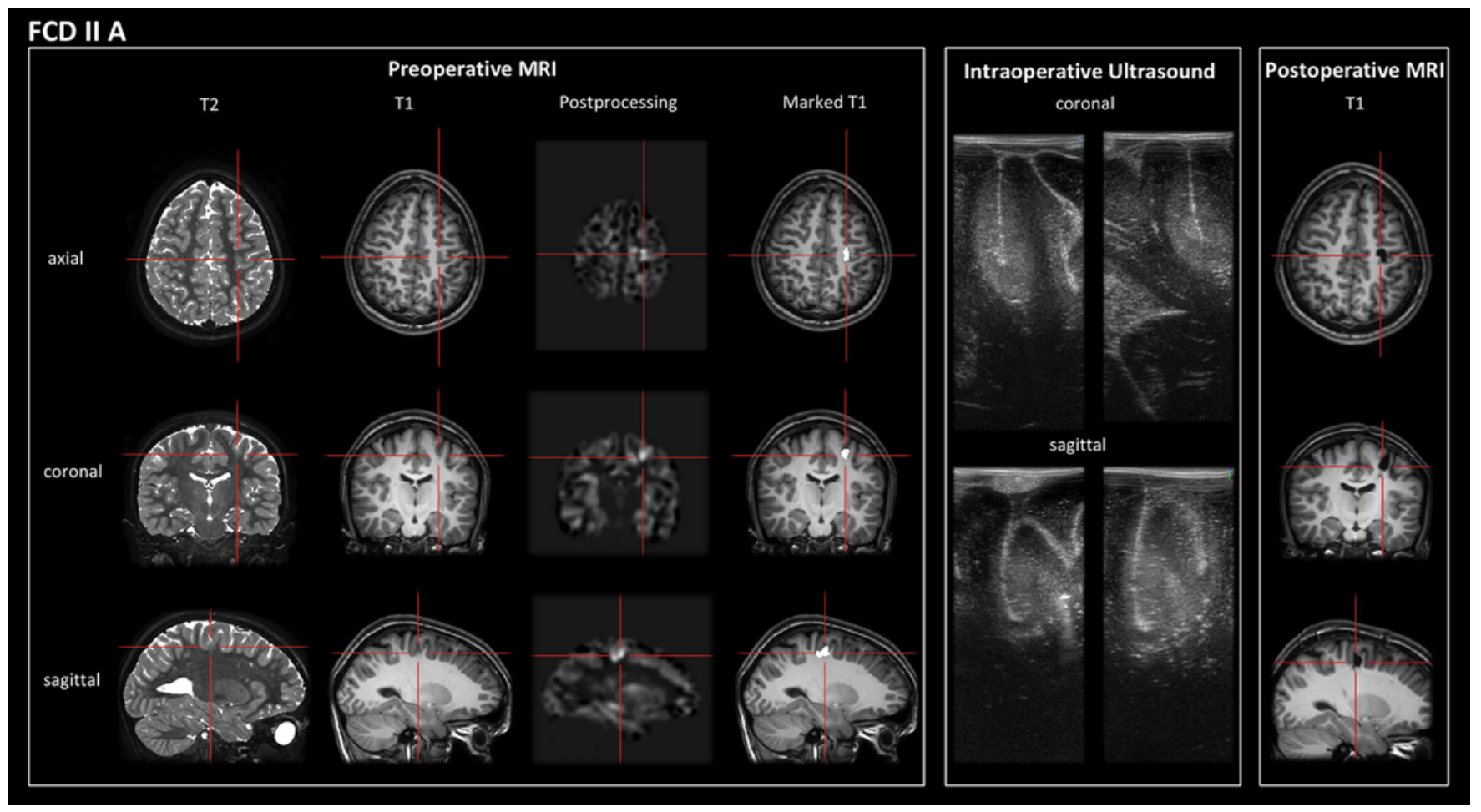

FIG. 2. Examples of pre-, intra-, and postoperative imaging in a patient with type IIA FCD in the depth of the superior precentral sulcus. Preoperative MRI with postprocessing (example sequence shown) revealed subtle cortical and subcortical T2-weighted and FLAIR hyperintensity, discrete increase in cortical thickness, and blurring of the gray-white-matter junction. On IOUS, this area suspicious for FCD could be easily identified by the marked hyperechogenicity of a thickened cortex, hyperechogenic, blurred gray-white-matter junction, and increased signal of the adjacent subcortical white matter, with high topographical-morphological correlation to preoperative imaging. Sharp demarcation of FCD borders by IOUS allowed us to perform complete resection without residual dysplasia on coregistration between pre- and postoperative MRI.

\section{Intraoperative and Immediate Postoperative Course}

The mean duration of the surgical procedure was 226 minutes (SD 54.5 minutes, median 210 minutes, range 165-355 minutes). No surgical complications such as intra- or postoperative hematoma, stroke, or wound infection were noted. Fourteen patients had an uneventful postoperative course, whereas 1 patient suffered from mild postoperative paresis and hyposensibility in the left lower extremity after removal of a right postcentral FCD adjacent to the central sulcus. This neurological deficit was considerably improved at last follow-up.

\section{Follow-Up}

At last follow-up (between 18 and 60 months-mean 35.9, SD 11.3, median 35 months), 10 of 15 (67\%) patients were completely seizure free (Engel IA) (Table 3), 1 had only simple partial nondisabling seizures, and 3 more had rare disabling seizures (Engel II). The patient with nonspecific gliosis showed no worthwhile improvement (Engel IVB).

Seizure outcome varied considerably between different FCD types. Two of the 3 patients with FCD type I were completely seizure free after surgery (Engel IA), whereas the other one had only simple partial nondisabling seizures after surgery (Engel IB). Residual dysplastic cortex at the resection border was identified in the 2 patients with FCD type I (1 with FCD type IA, 1 with FCD type IB) who achieved seizure freedom. Eight of the 11 patients with FCD type II were completely seizure free after surgery (Engel IA), and the other 3 had rare disabling seizures (Engel II). No residual dysplastic cortex was identified in any of the patients with FCD type II.

\section{Discussion}

To the best of our knowledge, this is the largest case series of IOUS utilization in epilepsy surgery, particularly in the context of FCD-associated drug-resistant epilepsy. The size of our cohort enables us to draw some reliable conclusions regarding the feasibility and added value of IOUS application in the resection of FCD.

FCD is one of the main underlying substrates in drugresistant partial epilepsy, especially in children. ${ }^{5}$ Resection is the most promising therapeutic option, with incomplete resection of the dysplastic cortex posing a major risk for seizure recurrence. ${ }^{12,17,26,29}$ Therefore, the optimal pre- and intraoperative definition of the lesion border and its resection are the key for surgical success. Dedicated MRI sequences, including MRI postprocessing techniques, markedly improved preoperative detection. However, intraoperative tools to guide FCD resection are limited. Direct visual and tactile information is generally poor in the case of FCD compared to other lesions such as brain tumors. ${ }^{33,35}$ Furthermore, neuronavigation accuracy is limited by its low overall resolution and by the risk of brain shift. ${ }^{1,11,14,33,35}$ 


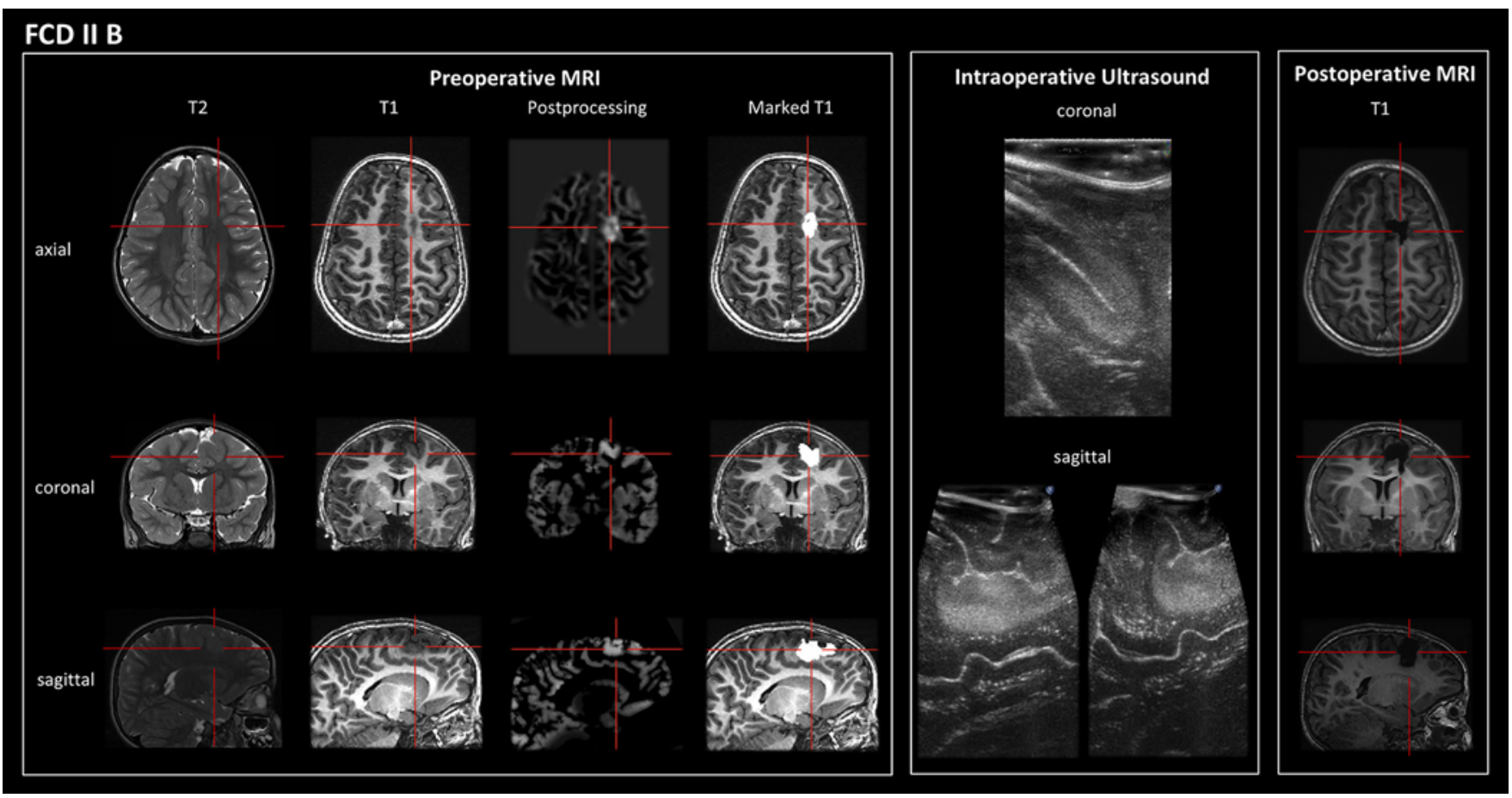

FIG. 3. Examples of pre-, intra-, and postoperative imaging in a patient with type IIB FCD of the superior frontal gyrus in the region of the medial sulcus. Preoperative MRI with postprocessing (example sequence shown) revealed cortical and subcortical T2-weighted and FLAIR hyperintensity, marked increase in cortical thickness, and pronounced blurring of the gray-white-matter junction. With high topographical-morphological correlation to preoperative imaging, IOUS revealed an area of heavily increased cortical thickness and marked hyperechogenicity in the cortex, at both the gray-white-matter junction and subcortically. Lesion borders could be clearly identified. Coregistration between pre- and postoperative MRI showed no residual dysplasia.

Intraoperative MRI has been reported to be useful, ${ }^{7,46}$ but its application is restricted by the limited availability, high costs, associated delay of surgery, low resolution, ${ }^{38}$ and absence of postprocessing techniques. Even though ECoG is of high value during FCD resection, it gives only 2D functional-and not anatomical-information, with a variable relation to the dysplastic region..$^{34}$ IOUS, on the contrary, is widely available and inexpensive. It has the advantage of being safe as well as quickly and repetitively applicable. IOUS interrupts the surgical procedure for only some seconds, in comparison to other intraoperative techniques such as acquisition of intraoperative MRI. It delivers realtime information, independent of brain shift, and provides 3D lesion visualization. Despite the many advantages of IOUS, only 3 studies, each reporting $1-5$ cases, $, 33,35,36$ have addressed the use of IOUS for FCD delineation in epilepsy surgery (Table 1).

Our study confirms the added value of IOUS for FCD detection, identification, and delineation. Sonographic visualization showed high correlation with preoperative MRI, including advanced postprocessing techniques. IOUS resolution was superior to MRI in all cases. Furthermore, sonographic features were shown to correlate to the underlying histopathological findings. First, this allows for a differentiation between FCD and non-FCD lesions (e.g., gliosis, characterized by diffuse nonorganized hyperechogenicity), which may have a direct impact on surgical strategy (e.g., intended extent or completeness of resection). Second, IOUS allows surgeons to estimate the underlying FCD type, due to the strong correlation between the se- verity of signal abnormalities and the degree of dysplasia (sonographic abnormalities increase continuously in the following order: FCD IA/IB, FCD IC, FCD IIA, FCD IIB). FCD type I, histologically defined by isolated dyslamination, showed only discrete cortical hyperechogenicity and blurring of the gray-white-matter junction. These features were similar in FCD type IA and IB, whereas they were more pronounced in the case of FCD type IC. This is in line with the histological properties of FCD types IA and IB, which are characterized by isolated radial or tangential dyslamination, whereas type IC combines these characteristics. Type II FCDs, histologically defined by the additional presence of dysmorphic neurons within the dysplastic area, showed more extensive sonographic abnormalities than with FCD I. The cortex appeared thickened and more hyperechogenic, the blurring of the gray-white-matter junction was more obvious, and there was some degree of subcortical hyperechogenicity. FCD IIB lesions (i.e., those containing balloon cells) displayed these features in an even more pronounced manner than type IIA. The clear sonographic visualization of FCD type II allowed for sharp demarcation of lesion borders in every case. Our results are in line with the reports from Miller and colleagues ${ }^{35,36}$ and Lee et al. ${ }^{33}$ Miller et al. described 3 cases of FCD type IIB characterized by marked hyperechogenicity and 1 of FCD type IA with faint hyperechogenicity. In contrast to our results, sonographic visualization was not possible in an FCD type IB in this study. ${ }^{36}$ The case of FCD IIA reported by Lee et al. was sonographically characterized by homogeneous hyperechogenicity. 


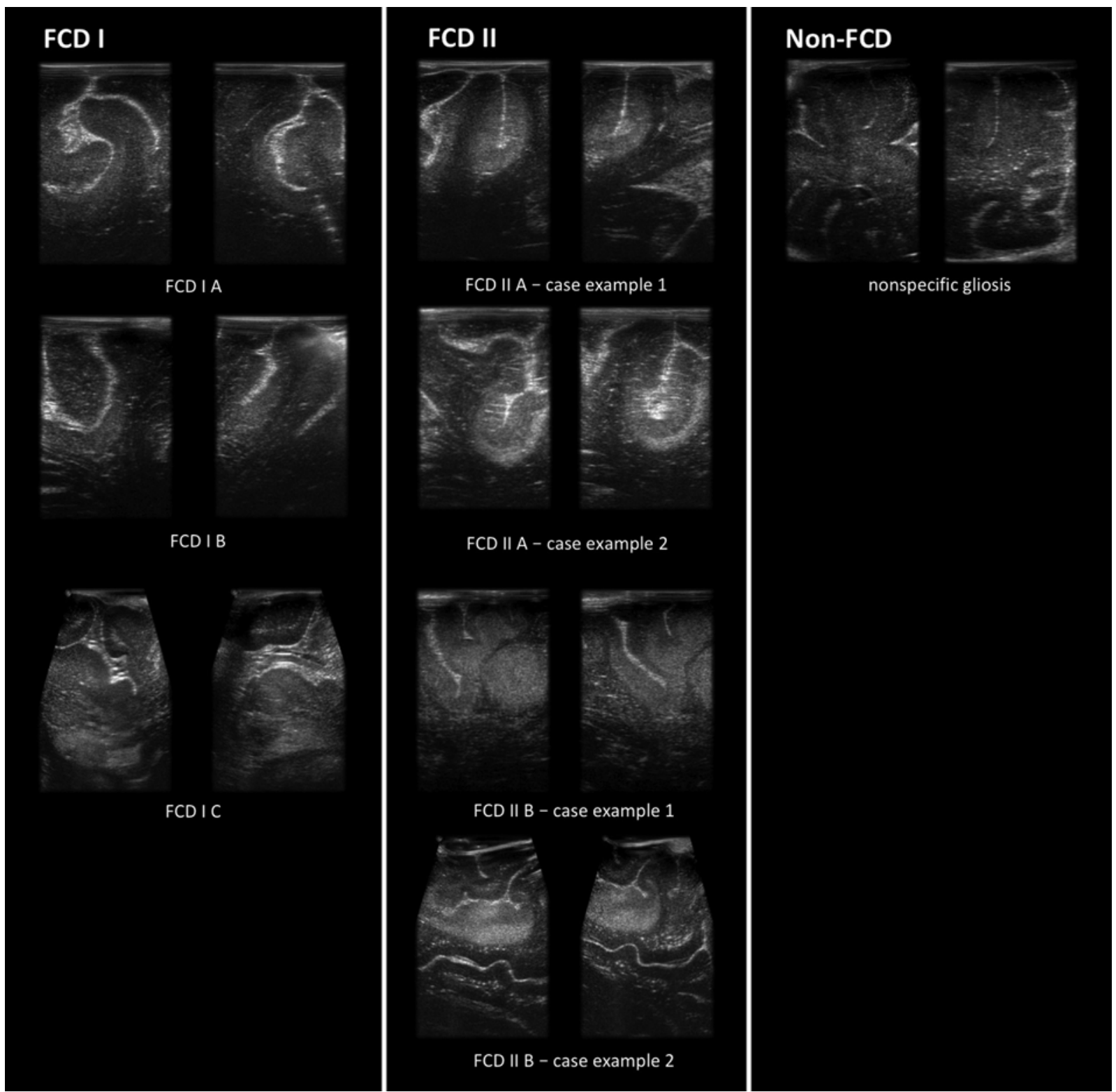

FIG. 4. Examples showing sonographic morphology of the different FCD subtypes versus nonspecific gliosis.

In our study, the extent of resection correlated with the demarcation of lesion margins on IOUS, which again matched the degree of dysplasia. IOUS allowed for complete resection in all FCD type II cases, whereas there was some marginal residual dysplasia in 2 cases (FCD IA and IB). Reported postsurgical seizure freedom rates in patients with FCD range from $33 \%$ to $90 \%$. $^{9,10,12,15,21,22,47-49,51}$ The size and heterogeneity of our cohort does not allow for definitive statements regarding seizure outcome. However, the $71 \%$ Engel IA outcome is particularly favorable. Surgery in FCD type II has previously been associated with superior seizure outcome as compared to FCD type I, 4,44,45 highlighting the challenges in the surgical approach for FCD type I. The inferior postsurgical outcome in patients with FCD type I-without IOUS - may be explained by the more difficult visualization with MRI, because cortical cellular density is only minimally altered in these FCD types. ${ }^{39}$ Patients with FCD type I in our series, however, had a favorable seizure outcome, despite the residual dysplasia. One possible explanation is that IOUS, by its high resolution, allows the surgeon to visualize and resect the regions of biologically relevant dysplasia in FCD type I, balancing the outcome between the different FCD subtypes.

This study shows the high potential of IOUS as a readily available neurosurgical tool in surgery for FCD-associated drug-resistant epilepsy, due to the excellent morphological resolution and close relation of the sonographic features to the histological degree of dysplasia. However, our findings should be interpreted with care, considering the following limitations. First, despite the reported higher resolution of IOUS, the role of MRI-and especially of postprocessing techniques-remains important and MRI cannot be replaced by IOUS. MRI is still the modality guiding 
the presurgical evaluation by identifying the potential epileptogenic lesion and determining the extent of resection. Second, IOUS interpretation remains subjective, and is highly dependent on the judgment and the experience of the investigator as well as on the apparatus and its settings. Third, the histopathological limitations of the 2011 classification $^{3}$ should be mentioned: the differentiation of FCD I subtypes is increasingly considered arbitrary; 39 they reportedly lack a clinical, imaging, or molecular phenotype. Despite the fact that the detected discrete sonographic differences between type IA/IB and IC represent such an imaging phenotype, the relevance of this differentiation remains unclear. The subdivision of FCD II into IIA and IIB has also been questioned. ${ }^{39}$ Sonographically, we identified these subtypes as different entities; however, the main distinction clearly remains between types I and II. Finally, limitations are posed by the small size of our cohort, which includes many different histological subtypes, precluding statistical analysis. Further research in larger cohorts and a prospective randomized design will be needed to make clear statements regarding the prognostic value of IOUS and its effect on residual dysplasia and seizure outcome.

\section{Conclusions}

This is the largest study to date investigating the yield of IOUS in surgery for FCD-associated drug-resistant epilepsy. Morphological features identified by IOUS show a high correlation with advanced preoperative imaging and may provide a superior resolution in some cases. The distinct sonographic features allow for the intraoperative distinction between FCD and non-FCD lesions as well as between the different histological subtypes of FCD. The sharpness of sonographic demarcation depends on the underlying degree of dysplasia. IOUS facilitates the completeness of resection by clearly delineating the dysplastic area.

\section{Acknowledgments}

We kindly thank the Prof. Dr. I. Blümcke and his team from the European FCD Reference Center in Erlangen for histopathological reference analysis.

\section{References}

1. Albert GW, Dahdaleh NS, Reddy C, Hansen DR, Vogel TW, Kawasaki H, et al: Postoperative radiographic findings in patients undergoing intracranial electrode monitoring for medically refractory epilepsy. J Neurosurg 112:449-454, 2010

2. Barkovich AJ, Guerrini R, Kuzniecky RI, Jackson GD, Dobyns WB: A developmental and genetic classification for malformations of cortical development: update 2012. Brain 135:1348-1369, 2012

3. Blümcke I, Spreafico R: An international consensus classification for focal cortical dysplasias. Lancet Neurol 10:26-27, 2011

4. Blumcke I, Spreafico R, Haaker G, Coras R, Kobow K, Bien $\mathrm{CG}$, et al: Histopathological findings in brain tissue obtained during epilepsy surgery. N Engl J Med 377:1648-1656, 2017

5. Blümcke I, Thom M, Aronica E, Armstrong DD, Vinters HV, Palmini A, et al: The clinicopathologic spectrum of focal cortical dysplasias: a consensus classification proposed by an ad hoc Task Force of the ILAE Diagnostic Methods Commission. Epilepsia 52:158-174, 2011

6. Boonyapisit K, Najm I, Klem G, Ying Z, Burrier C, LaPresto E, et al: Epileptogenicity of focal malformations due to abnormal cortical development: direct electrocorticographichistopathologic correlations. Epilepsia 44:69-76, 2003

7. Buchfelder M, Fahlbusch R, Ganslandt O, Stefan H, Nimsky C: Use of intraoperative magnetic resonance imaging in tailored temporal lobe surgeries for epilepsy. Epilepsia 43:864-873, 2002

8. Burkhardt JK, Serra C, Neidert MC, Woernle CM, Fierstra J, Regli L, et al: High-frequency intra-operative ultrasoundguided surgery of superficial intra-cerebral lesions via a single-burr-hole approach. Ultrasound Med Biol 40:1469-1475, 2014

9. Chassoux F, Landré E, Mellerio C, Turak B, Mann MW, Daumas-Duport $\mathrm{C}$, et al: Type II focal cortical dysplasia: electroclinical phenotype and surgical outcome related to imaging. Epilepsia 53:349-358, 2012

10. Chung CK, Lee SK, Kim KJ: Surgical outcome of epilepsy caused by cortical dysplasia. Epilepsia 46 (Suppl 1):25-29, 2005

11. Coenen VA, Krings T, Weidemann J, Hans FJ, Reinacher P, Gilsbach JM, et al: Sequential visualization of brain and fiber tract deformation during intracranial surgery with threedimensional ultrasound: an approach to evaluate the effect of brain shift. Neurosurgery 56 (1 Suppl):133-141, 2005

12. Cohen-Gadol AA, Ozduman K, Bronen RA, Kim JH, Spencer DD: Long-term outcome after epilepsy surgery for focal cortical dysplasia. J Neurosurg 101:55-65, 2004

13. Colombo N, Tassi L, Galli C, Citterio A, Lo Russo G, Scialfa G, et al: Focal cortical dysplasias: MR imaging, histopathologic, and clinical correlations in surgically treated patients with epilepsy. AJNR Am J Neuroradiol 24:724733,2003

14. Dorward NL, Alberti O, Velani B, Gerritsen FA, Harkness WF, Kitchen ND, et al: Postimaging brain distortion: magnitude, correlates, and impact on neuronavigation. J Neurosurg 88:656-662, 1998

15. Edwards JC, Wyllie E, Ruggeri PM, Bingaman W, Lüders H, Kotagal P, et al: Seizure outcome after surgery for epilepsy due to malformation of cortical development. Neurology 55:1110-1114, 2000

16. Engel J Jr, Van Ness PC, Rasmussen TB, Ojemann LM: Outcome with respect to epileptic seizures, in Engel J Jr (ed): Surgical Treatment of the Epilepsies, ed 2. New York: Raven Press, 1993, pp 609-621

17. Fauser S, Essang C, Altenmüller DM, Staack AM, Steinhoff BJ, Strobl K, et al: Long-term seizure outcome in 211 patients with focal cortical dysplasia. Epilepsia 56:66-76, 2015

18. Fauser S, Huppertz HJ, Bast T, Strobl K, Pantazis G, Altenmueller DM, et al: Clinical characteristics in focal cortical dysplasia: a retrospective evaluation in a series of 120 patients. Brain 129:1907-1916, 2006

19. Fisher RS: The New Classification of Seizures by the International League Against Epilepsy 2017. Curr Neurol Neurosci Rep 17:48, 2017

20. Focke NK, Symms MR, Burdett JL, Duncan JS: Voxel-based analysis of whole brain FLAIR at 3T detects focal cortical dysplasia. Epilepsia 49:786-793, 2008

21. Guerrini R, Duchowny M, Jayakar P, Krsek P, Kahane P, Tassi L, et al: Diagnostic methods and treatment options for focal cortical dysplasia. Epilepsia 56:1669-1686, 2015

22. Harvey AS, Mandelstam SA, Maixner WJ, Leventer RJ, Semmelroch M, MacGregor D, et al: The surgically remediable syndrome of epilepsy associated with bottom-of-sulcus dysplasia. Neurology 84:2021-2028, 2015

23. House PM, Lanz M, Holst B, Martens T, Stodieck S, Huppertz HJ: Comparison of morphometric analysis based on T1- 
and T2-weighted MRI data for visualization of focal cortical dysplasia. Epilepsy Res 106:403-409, 2013

24. Huppertz HJ, Grimm C, Fauser S, Kassubek J, Mader I, Hochmuth A, et al: Enhanced visualization of blurred graywhite matter junctions in focal cortical dysplasia by voxelbased 3D MRI analysis. Epilepsy Res 67:35-50, 2005

25. Huppertz HJ, Kassubek J, Altenmüller DM, Breyer T, Fauser S: Automatic curvilinear reformatting of three-dimensional MRI data of the cerebral cortex. Neuroimage 39:80-86, 2008

26. Kim DW, Lee SK, Chu K, Park KI, Lee SY, Lee CH, et al: Predictors of surgical outcome and pathologic considerations in focal cortical dysplasia. Neurology 72:211-216, 2009

27. Kim YH, Kang HC, Kim DS, Kim SH, Shim KW, Kim HD, et al: Neuroimaging in identifying focal cortical dysplasia and prognostic factors in pediatric and adolescent epilepsy surgery. Epilepsia 52:722-727, 2011

28. Kovalev D, Spreer J, Honegger J, Zentner J, Schulze-Bonhage A, Huppertz HJ: Rapid and fully automated visualization of subdural electrodes in the presurgical evaluation of epilepsy patients. AJNR Am J Neuroradiol 26:1078-1083, 2005

29. Krsek P, Maton B, Jayakar P, Dean P, Korman B, Rey G, et al: Incomplete resection of focal cortical dysplasia is the main predictor of poor postsurgical outcome. Neurology 72:217-223, 2009

30. Krsek P, Pieper T, Karlmeier A, Hildebrandt M, Kolodziejczyk D, Winkler P, et al: Different presurgical characteristics and seizure outcomes in children with focal cortical dysplasia type I or II. Epilepsia 50:125-137, 2009

31. Kwan P, Arzimanoglou A, Berg AT, Brodie MJ, Allen Hauser W, Mathern G, et al: Definition of drug resistant epilepsy: consensus proposal by the ad hoc Task Force of the ILAE Commission on Therapeutic Strategies. Epilepsia 51:10691077,2010

32. Kwan P, Brodie MJ: Early identification of refractory epilepsy. N Engl J Med 342:314-319, 2000

33. Lee CC, Lin CF, Yu HY, Hung SC, Shih YH, Hsu SPC: Applications of intraoperative ultrasound in epilepsy surgery for focal cortical dysplasia. J Med Ultrasound 22:43-46, 2014

34. Lüders H, Awad I, Burgess R, Wyllie E, Van Ness P: Subdural electrodes in the presurgical evaluation for surgery of epilepsy. Epilepsy Res Suppl 5:147-156, 1992

35. Miller D, Knake S, Bauer S, Krakow K, Pagenstecher A, Sure $\mathrm{U}$, et al: Intraoperative ultrasound to define focal cortical dysplasia in epilepsy surgery. Epilepsia 49:156-158, 2008

36. Miller D, Knake S, Menzler K, Krakow K, Rosenow F, Sure $\mathrm{U}$ : Intraoperative ultrasound in malformations of cortical development. Ultraschall Med 32 (Suppl 2):E69-E74, 2011

37. Mischel PS, Nguyen LP, Vinters HV: Cerebral cortical dysplasia associated with pediatric epilepsy. Review of neuropathologic features and proposal for a grading system. J Neuropathol Exp Neurol 54:137-153, 1995

38. Mittal S, Black PM: Intraoperative magnetic resonance imaging in neurosurgery: the Brigham concept. Acta Neurochir Suppl 98:77-86, 2006

39. Najm IM, Sarnat HB, Blümcke I: Review: The international consensus classification of focal cortical dysplasia-a critical update 2018. Neuropathol Appl Neurobiol 44:18-31, 2018

40. Palmini A, Najm I, Avanzini G, Babb T, Guerrini R, Foldvary-Schaefer $\mathrm{N}$, et al: Terminology and classification of the cortical dysplasias. Neurology 62 (6 Suppl 3):S2-S8, 2004

41. Ramantani G, Kadish NE, Mayer H, Anastasopoulos C, Wagner K, Reuner G, et al: Frontal lobe epilepsy surgery in childhood and adolescence: predictors of long-term seizure freedom, overall cognitive and adaptive functioning. Neurosurgery 83:93-103, 2018

42. Ramantani G, Kadish NE, Strobl K, Brandt A, Stathi A, Mayer H, et al: Seizure and cognitive outcomes of epilepsy surgery in infancy and early childhood. Eur J Paediatr Neurol 17:498-506, 2013

43. Ramantani G, Stathi A, Brandt A, Strobl K, Schubert-Bast $\mathrm{S}$, Wiegand G, et al: Posterior cortex epilepsy surgery in childhood and adolescence: predictors of long-term seizure outcome. Epilepsia 58:412-419, 2017

44. Ramírez-Molina JL, Di Giacomo R, Mariani V, Deleo F, Cardinale F, Uscátegui-Daccarett AM, et al: Surgical outcomes in two different age groups with focal cortical dysplasia type II: any real difference? Epilepsy Behav 70 (Pt A):45-49, 2017

45. Rowland NC, Englot DJ, Cage TA, Sughrue ME, Barbaro NM, Chang EF: A meta-analysis of predictors of seizure freedom in the surgical management of focal cortical dysplasia. J Neurosurg 116:1035-1041, 2012

46. Schwartz TH, Marks D, Pak J, Hill J, Mandelbaum DE, Holodny AI, et al: Standardization of amygdalohippocampectomy with intraoperative magnetic resonance imaging: preliminary experience. Epilepsia 43:430-436, 2002

47. Siegel AM, Cascino GD, Meyer FB, Marsh WR, Scheithauer BW, Sharbrough FW: Surgical outcome and predictive factors in adult patients with intractable epilepsy and focal cortical dysplasia. Acta Neurol Scand 113:65-71, 2006

48. Sisodiya SM: Surgery for malformations of cortical development causing epilepsy. Brain 123 (Pt 6):1075-1091, 2000

49. Tassi L, Colombo N, Garbelli R, Francione S, Lo Russo G, Mai R, et al: Focal cortical dysplasia: neuropathological subtypes, EEG, neuroimaging and surgical outcome. Brain 125:1719-1732, 2002

50. Taylor DC, Falconer MA, Bruton CJ, Corsellis JA: Focal dysplasia of the cerebral cortex in epilepsy. J Neurol Neurosurg Psychiatry 34:369-387, 1971

51. Tripathi M, Singh MS, Padma MV, Gaikwad S, Bal CS, Tripathi M, et al: Surgical outcome of cortical dysplasias presenting with chronic intractable epilepsy: a 10-year experience. Neurol India 56:138-143, 2008

52. Wagner J, Weber B, Urbach H, Elger CE, Huppertz HJ: Morphometric MRI analysis improves detection of focal cortical dysplasia type II. Brain 134:2844-2854, 2011

53. Widdess-Walsh P, Kellinghaus C, Jeha L, Kotagal P, Prayson $\mathrm{R}$, Bingaman W, et al: Electro-clinical and imaging characteristics of focal cortical dysplasia: correlation with pathological subtypes. Epilepsy Res 67:25-33, 2005

54. World Health Organization: Epilepsy fact sheet. WHO.int. February 8, 2018 (http://www.who.int/news-room/fact-sheets/ detail/epilepsy) [Accessed July 11, 2018]

\section{Disclosures}

The authors report no conflict of interest concerning the materials or methods used in this study or the findings specified in this paper.

\section{Author Contributions}

Conception and design: Akeret, Krayenbühl. Acquisition of data: Akeret. Analysis and interpretation of data: Akeret, Bellut. Drafting the article: Akeret. Critically revising the article: Bellut, Huppertz, Ramantani, König, Serra, Regli, Krayenbühl. Reviewed submitted version of manuscript: Bellut, Ramantani, Krayenbühl. Statistical analysis: Akeret. Administrative/technical/material support: Akeret. Study supervision: Krayenbühl.

\section{Correspondence}

Kevin Akeret: University Hospital Zurich, Switzerland. kevin. akeret@gmx.ch. 